\title{
Dynamic Voltage/Var Sensitivity Approach for Improving Fault-induced Voltage Delayed Recovery Problems
}

\author{
Zhang Yue ${ }^{1, a}$, Li Xiaoming ${ }^{2, b}$ \\ 1.Electric Power Research Institute of Guangdong Power Grid Corporation, Guangzhou 510080, \\ Guangdong Province, China \\ ${ }^{2}$ School of Electrical Engineering, Northeast Dianli University, Jilin 132012,Jilin Province, China \\ ayuetju@163.com, ${ }^{\mathrm{b}} 13732968216 @ 163 . c o m$
}

Keywords: key bus; Integral mapping; sensitivity factor of dynamic voltage/var; voltage delayed recovery.

\begin{abstract}
A dynamic sensitivity method is proposed to evaluate the impact of generators' reactive power on the recovery of key bus voltage in a system post-fault dynamic duration to improve the problem of delayed recovery voltage. Voltage delayed recovery existed in actual power grid is simulated on Guangdong power grid of 2013 to verify the correctness and effectiveness of the method. It is significant to solve the problems of selecting the plant units involved in the reactive power control for shortening the recovery time of key bus voltage.
\end{abstract}

\section{Introduction}

In recent years, due to the growing power demand, a hybrid AC/DC power grid is built, which is of ultra-high voltage, long distance and large capacity. With the economic and environmental constraints, power system operation draws near its limits, and the problem of voltage stability is more and more prominent [1]-[4].

Voltage is one of the important indices in evaluation power quality. Maintaining normal voltage level is an important guarantee for the safe operation of the power system. Voltage levels is closely related to reactive power balance, so that reasonably regulating of reactive power source is an important measure to keep system stability and voltage levels. As dynamic reactive power in the power system, generation is able to adjust reactive power fast and automatically in a large range without the additional investment[5].

This paper proposes a dynamic voltage/reactive sensitivity index based on integral mapping. It is of great significance to solve the problem of voltage recovery time long. applying PSD-BPA analysis toolkits to simulate bus voltage for Guangdong operation mode, it is demonstrated that the solution is correct and availability[6-7].

\section{Analysis of Voltage Recovery after Failure}

Currently, the majority of the literature are used voltage-dropping magnitude and its duration as described feature quantity. In the IEEE standards about power quality, the feature of voltage dropping is that the voltage amplitude is in the range between 0.1p.u. and 0.9p.u., its duration between 0.01 to 1 seconds[8]. To ensure the safe operation, many power companies at home and 
abroad set special requirements for voltage recovery rate after disturbances. State grid corporation in the electric power system safe and stable computing specifications, also has specific rules about voltage delayed recovery .

In this section, Guangdong Power Grid in 2013 simulation results show the system exists voltage delayed problems under a three-phase short circuit fault. The failure of (hereafter referred to as fault-A): $500 \mathrm{kV}$ Beijiao side of Beihuajia line failed in 0.1s (5 cycles), the switch broke in Huadu side on 0.2s, while the single-phase switch missed operation in Beijiao side; Beihuajia line is cut on 0.35s. Near Beijiao station, 220kV Luming、Luochong、Tianhe substation existed the problem of voltage delayed recovery, the simulation profiles shown in Figure 1

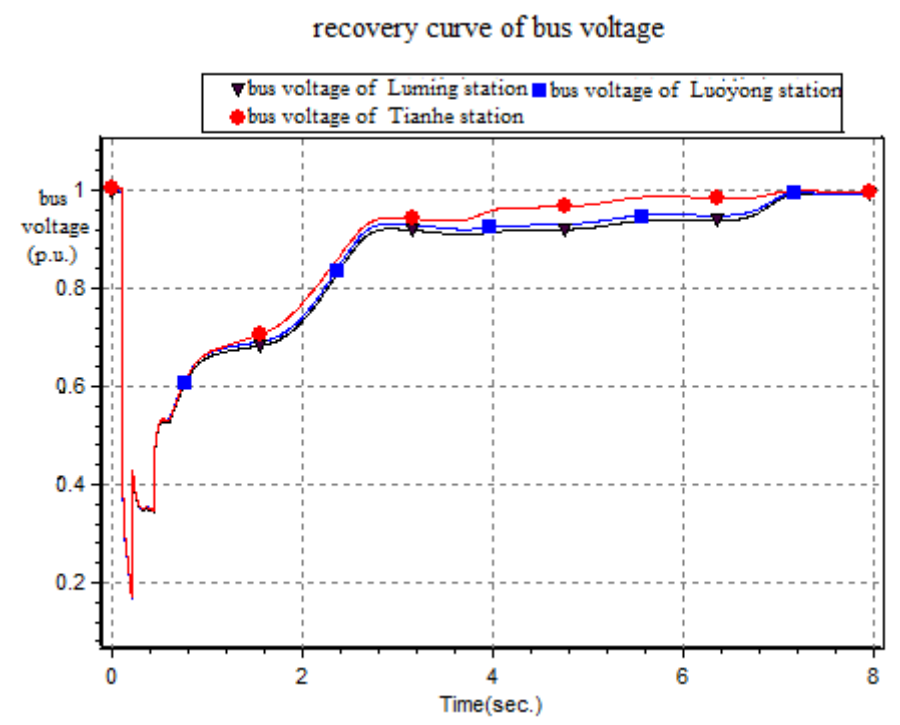

Fig 1. voltage profiles of $220 \mathrm{kV}$ Luming, Luochong, Tianhe stations for fault A

Figure 1 shows bus voltage in Luming station kept low voltage for a long time after the failure, down below 0.75p.u. duration of up to 79 cycles, and down below 0.95p.u. duration of up to 315 cycles. According to the guidelines for power system security and stability in our country and the southern power grid security and stability guideline, the bus voltage regulation is beyond the guideline standard $[9,10]$ of "transient and dynamic system is the central point in the process of bus voltage dropping below 0.75 p.u. duration less than $1 \mathrm{s"}$.

\section{Dynamic Response Characteristic of Generation Reactive Power}

For fault A, this section took Guangxu power plant as an example, analyzing the effects of generation excitation reference voltage controlling on dynamic reactive power output of the generation. The generations in Guangxu power plant, respectively carried out with/without 2\% reference voltage step excitation controlling, reactive power output curves in two cases after the fault A, as shown in Figure 2.

Figure 2 shows that $2 \%$ reference voltage step excitation controlling by the unit in Guangxu power plant can rapid increase generation reactive power output at 2.2 seconds. The simulation results indicate the effects of reference voltage step excitation controlling on reactive power output are significant and the controlling is effective for improving voltage delayed recovery. 


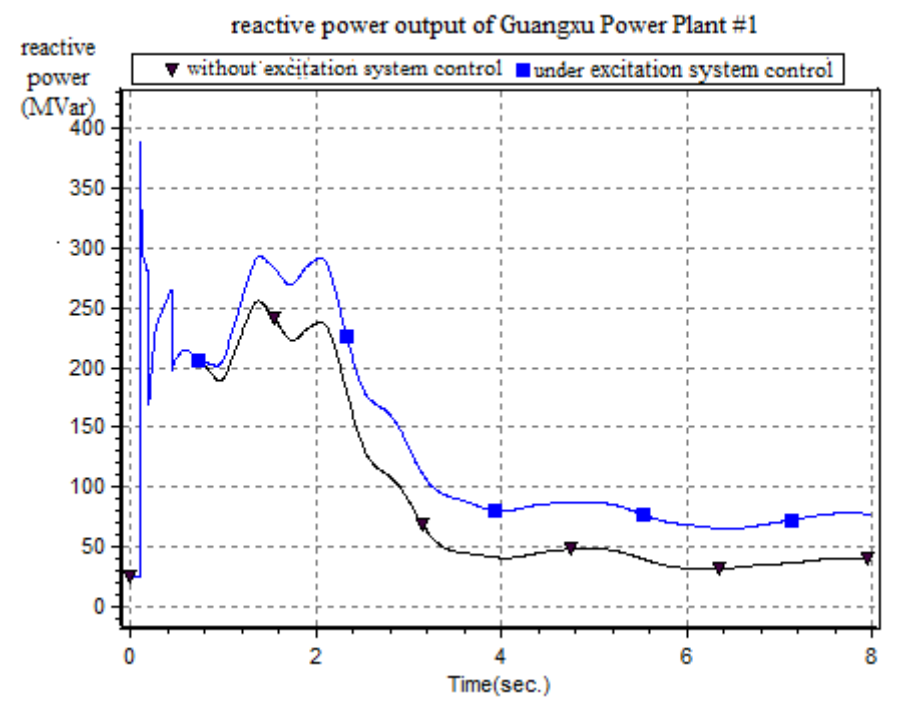

Fig 2 . The BPA simulation curves of reactive power output of \#1 generator in Guangxu Power Plant for fault A

\section{Dynamic Voltage / Reactive Sensitivity Model}

\subsection{Integral Mapping Model for Voltage Increment Measure}

When the system happened large disturbance or failure of certain key substation bus voltage dropping occurs, a power plant generation will achieve rapid self-starting excitation controlling, in order to increase reactive power output. Assuming the measure of the key bus l voltage increment is $\Delta \bar{V}_{i i}$ during the failure, according to equation (1) is calculated.

$$
\Delta \bar{V}_{l i}=\bar{V}_{l i}^{(2)}-\bar{V}_{l i}^{(1)}=\left(\int_{t_{0}}^{t_{w}} V_{l i}^{(2)}(t) d t-\int_{t_{0}}^{t_{w}} V_{l i}^{(1)}(t) d t\right) /\left(t_{w}-t_{0}\right)
$$

where, $V_{l i}^{(2)}(t)$ and $V_{l i}^{(1)}(t)$ respectively represent the bus 1 voltage of moment $t$ after the plants unit $i$ excitation voltage reference implementation $1 \%$ step $2 \%$ step; $t_{0}$ and $t_{w}$ respectively represent the recovery time of failure and voltage back to $0.75 \mathrm{pu}$; Apparently $\Delta \bar{v}_{l i}$ indicates that the plants unit $i$ is controlled by implementing $1 \%$ step $2 \%$ step to implement the control bus voltage value increment. Discretization of $\Delta \bar{V}_{i i}$ by formula (2).

$$
\Delta \bar{V}_{l i}=\left(\sum _ { k = 0 } ^ { N } \left(V_{l i}^{(2)}\left(t_{k}\right)-\sum_{k=1}^{N}\left(V_{l i}^{(1)}\left(t_{k}\right)\right) \Delta t /\left(t_{w}-t_{0}\right)\right.\right.
$$

where, $V_{l i}^{(2)}\left(t_{k}\right)$ and $V_{l i}^{(1)}\left(t_{k}\right)$ respectively express the key bus 1 voltage value of $t_{k}$ moment after the plants unit $i$ excitation voltage reference implementation and $1 \%$ step $2 \%$ step. $N$ expressed failure recovery time intervals.

\subsection{Integral Mapping Model for Increment Measure of Generation Reactive Power}

In the dynamic process, voltage/reactive sensitivity model not only calculate the key bus voltage's increment measure, but also evaluate reactive power increment measure after controlling excitation reference voltage step by the reactive power output of power plant unit itself, it is able to be represented below.

$$
\Delta \bar{Q}_{i}=\bar{Q}_{i}^{(2)}-\bar{Q}_{i}^{(1)}=\left(\int_{t_{0}}^{t_{w}} Q_{i}^{(2)}(t) d t-\int_{t_{0}}^{t_{w}} Q_{i}^{(1)}(t) d t\right) /\left(t_{w}-t_{0}\right)
$$


where, $Q_{i}^{(2)}(t)$ and $Q_{i}^{(1)}(t)$ correspond to the value of reactive power at the time after the unit is excited reference voltage $2 \%$ and $1 \%$ of the step control; $t_{0}$ and $t_{w}$ have the same meanings of formula (1). $\Delta \bar{Q}_{i}$ indicates that the plants $i$ unit is controlled by implementing $1 \%$ step $2 \%$ step to implement control the reactive power mean value of increment. Similar to formula (2), $\Delta \bar{V}_{l i}$ discrete calculation by the formula (4).

$$
\Delta \bar{Q}_{i}=\left(\sum _ { k = 1 } ^ { N } \left(Q_{i}^{(2)}\left(t_{k}\right)-\sum_{k=1}^{N}\left(Q_{i}^{(1)}\left(t_{k}\right)\right) \Delta t /\left(t_{w}-t_{0}\right)\right.\right.
$$

\subsection{Dynamic Voltage/Var Sensitivity}

Using the two definition of key bus l voltage's increment measure $\Delta \bar{V}_{l i}$ and corresponding reactive incremental $\Delta \bar{Q}_{i}$ measure. It is as shown in formula (5).

$$
\mu_{1 i}=\frac{\Delta \bar{V}_{l i}}{\Delta \bar{Q}_{i}}
$$

The dynamic voltage/var sensitivity index regards the recovery measure of the key bus voltage after disturbance as state variables, reactive power of the generation unites with the step controlling of the excitation reference voltage as control variables.

\section{Case Studies}

Fault A is selected to check the proposed approach. As shown in Fig.1, Near Beijiao station, 220kV Luming 、 Luochong、Tianhe substation existed the problem of voltage delayed recovery. The unit in Jiangzhongzhu area is only selected, achieving $2 \%$ and $1 \%$ excitation step controlling.

The step excitation control of power generating units around Jiangzhongzhu, respectively the corresponding results are shown in table 1.

Tab 1: For fault A, the corresponding results after excitation control

\begin{tabular}{cccc} 
Power plant & $\Delta \bar{V}_{l i}[\mathrm{kV}]$ & $\Delta \bar{Q}_{i}[\mathrm{Mvar}]$ & $\mu_{l i}$ \\
\hline Tonggu B & 0.0020 & 122.9451 & 0.0036 \\
Tonggu A & 0.0006 & 39.7659 & 0.0033 \\
Shuangshui & 0.0002 & 18.3513 & 0.0024 \\
Zhuhai B & 0.0010 & 96.8807 & 0.0023 \\
Yongan & 0.0001 & 10.2456 & 0.0021 \\
\hline
\end{tabular}

The numerical results between voltage reactive and power output of integral mapping sensitivity of power plant units (from largest to smallest) in $220 \mathrm{kV}$ Luming station, is shown in the 3rd column. The sensitivity data shows that for the fault A, Tonggu B sensitivity which is controlled by excitation based on the integral mapping is maximum, Tonggu A sensitivity followed. Sensitivity is defined by equation (5), for the fault A, Jiangzhongzhu area firstly launched a unit with larger sensitivity $\mu_{l i}$ joining excitation and reference voltage step control to make greater effect on decreasing the $220 \mathrm{kV}$ Luming station bus voltage recovery time.

The simulation results express that the implementation of the excitation controlling higher sensitivity five power plants, Luming station bus voltage recovery characteristics are of the best. The sensitivity of the simulation results shows that the proposed indicators can accurately reflect the effect of unit excitation controlling of bus voltage recovery, the selection of the excitation controlling of power units in improving key bus voltage recovery characteristics have accurate guidance. 


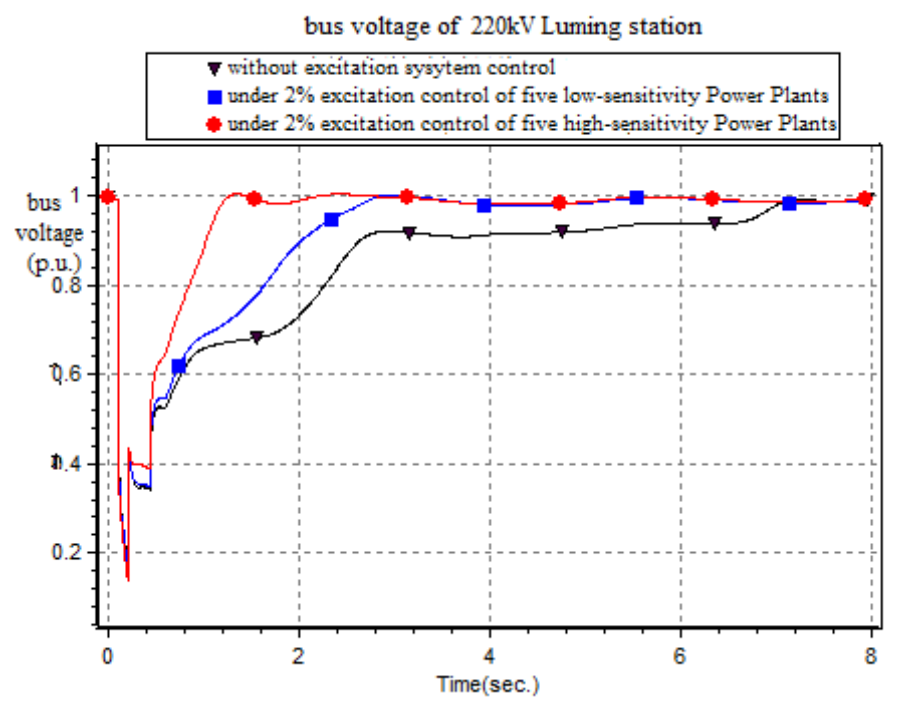

Fig 3. For fault A, 220kV Luming station voltage curve after excitation control of specified plants

\section{Conclusion}

This paper presents a dynamic voltage/var sensitivity approach based on integrating mapping, After actual power grid failure, reasonable choice involved in reactive power controlling of plant, it is of significance in improving key bus voltage recovery problem. It lies the theoretical foundation for analyzing the control strategy of key bus voltage recovery after disturbances on the issue.

\section{References}

[1] Su Yongchun, Cheng Shijie, Wen Jinyu.Analytic continuation of power system dynamic voltage stability analysis [J].Proceedings of the CSEE, 2007,27 (4): 9-14.

[2] Liu Yiqing, Chen Chaoying, Liang Lei, Liu Li.Review of power system dynamic voltage stability analysis methods [J].Proceedings of the Chinese Society of Universities for Electric Power System and its Automation, 2003,15 (1): 105-108.

[3] Tang Yong, Zhong Wuzhi, Sun Huadong, Lin Weifang, Yi Jun, Zhang Xin.Power system voltage stability study on the mechanism of [J].Power System Technology, 2010,34 (4): 24-29.

[4] Liu Daowei, Han Xueshan, Wang Yong, Wang Mengxia, Yun Chi-Ho.Online study and application of power system static stability region [J].Proceedings of the CSEE, 2009,29 (34): 42-49.

[5] An Jun, Mu Gang, Zheng Taiyi, Wang mingxing, Liu bailin, Jiang Xu.Improve voltage level of adjustment coefficients optimization strategy of generation excitation system [J].Power system automation, 2013,37 (23): 97-101.

[6] Sun Huadong, Zhou Xiaoxin, Li Ruomei.Static voltage stability analysis considering induction motor loads [J].Proceedings of the CSEE, 2005,25 (24): 1-6.

[7] IEEE Std 1159-1995,IEEE Recommended Practice for Monitoring Electric Power Quality[S].

[8] Q/GDW404-2010, national grid computing technology, the security and stability of specification [S].

[9] DL755-2001, guidelines for power system safe and stable [S].

[10] Q/CSG11004-2009, southern power grid safety and stability analysis of Guide [S]. 